\title{
Entrelacs
}

Cinéma et audiovisuel

$7 \mid 2009$

L'atelier

\section{L'atelier, ou le bureau du temps}

Tableau avec chutes, de Claudio Pazienza

Rose-Marie Godier

\section{(2) OpenEdition}

Journals

Édition électronique

URL : http://journals.openedition.org/entrelacs/176

DOI : $10.4000 /$ entrelacs. 176

ISSN : 2261-5482

Éditeur

Éditions Téraèdre

Édition imprimée

Date de publication : 1 mars 2009

Pagination : 23-31

ISBN : 978-2-912868-69-5

ISSN : 1266-7188

Référence électronique

Rose-Marie Godier, «L'atelier, ou le bureau du temps », Entrelacs [En ligne], 7 | 2009, mis en ligne le 01 août 2012, consulté le 22 avril 2019. URL : http://journals.openedition.org/entrelacs/176 ; DOI :

10.4000/entrelacs. 176

Ce document a été généré automatiquement le 22 avril 2019.

Tous droits réservés 


\title{
L'atelier, ou le bureau du temps
}

\author{
Tableau avec chutes, de Claudio Pazienza
}

Rose-Marie Godier

1 Parce qu'il est d'abord, et dès le premier plan, offre d'un regard, le film de Claudio Pazienza, Tableau avec chutes, est l'occasion d'une expérience singulière. C'est de cette expérience de spectateur que les lignes qui suivent voudraient rendre compte. Dans le foisonnement des images, des sons, des idées, des pensées que propose ce film - en réponse assurément à la passionnante richesse des tableaux de Bruegel - et puisqu'il faut bien partir d'un point pour s'engager dans ce voyage, nous choisirons de partir d'un détail placé au centre du film : l'étonnant Bureau du Temps dont l'entrée, nous dit-on, est interdite « sauf pour les nécessités du service ». Ici, l'entrée s'avère nécessaire car ce film, qui s'affiche de prime abord comme «Voyage dans un tableau de Pieter Bruegel, Paysage avec la chute d'Icare, peint vers 1555 quelque part en Belgique », entreprend de tisser entre elles de multiples lignes de temps.

2 Sa matière est d'emblée constituée de l'entrelacs, du montage de temps hétérogènes, puisque le film n'a pas pour but de nous montrer un tableau de la Renaissance flamande en s'en tenant à l'écart, c'est-à-dire à la simple surface, mais d'envisager dans quelle mesure, aujourd'hui, ce tableau nous regarde. Et, plus précisément, il s'agit ici, pour reprendre les mots de Walter Benjamin, de "rendre présentes les corrélations du passé », afin d'allumer «la mèche de l'explosif qui gît dans ce qui a été »'. C'est cette rencontre entre passé et présent que défend farouchement dans le film un Pazienza, muni d'énormes gants de boxe et prêt à tous les combats, devant le tableau des Musées royaux des Beauxarts de Bruxelles. Tableau avec chutes entend bien explorer les nécessités et surtout la fécondité de l'anachronisme - mais toute histoire de l'art, celle du moins qui ne traite pas d'objets morts ou de fossiles, ne laisse pas d'être elle-même une "discipline anachronique $»^{2}$.

3 Le prétexte du film serait donc cet impérieux regard sur le cinéaste d'aujourd'hui du peintre de naguère; le travail du film, et d'abord le processus de filmage, consistera à élucider la nature exacte de certaines affinités, emboîtements sensibles et secrètes tangentes. Le regard que portait Bruegel sur son temps était assez large pour embrasser à la fois les noces et les danses paysannes, les travaux des champs, les saisons, la chasse et 
la taille des arbres, les jeux d'enfants, mais aussi bien les villages calcinés, les hurlements des batailles, les fureurs du feu, la folie noire et les gibets, le triomphe de la mort. Sur la nature de ce regard, qu'Aldous Huxley a pu qualifier d' "anthropologique ", le film s'interroge, qui superpose un détail du Massacre des innocents à l'évocation du souvenir du duc d'Albe ; un détail du Repas de noces à celle des paysans affamés de ce temps ; un couple extrait de La Danse des paysans à l'hypothèse de chastes amoureux. Mais, plus avant, la question se déplace et expérimente le regard de Bruegel sur notre actualité : entremêlés d'images quotidiennes, Le Dénombrement de Bethléem accompagne l'arrivée en Belgique des parents de l'auteur ; les trois œufs tirés du Combat de Carnaval et Carême soulignent l'effort du gouvernement à ramener le déficit à trois pour cent ; une des " gueules subhumaines " ${ }^{4}$ de La Chute des anges rebelles est associée aux chômeurs, dont le nombre est, dit-on, toujours stable; les Deux singes enchaînés gardent la même valeur qu'au temps de Bruegel : la cœxistence entre Wallons et Flamands - l'ironie réside peut-être dans la constance de cette interprétation ${ }^{5}$.

Aussi bien Bruegel pratiquait-il lui-même l'anachronisme dans ces tableaux qui inscrivent Le Massacre des innocents ou Le Portement de croix dans la trame des travaux quotidiens de son siècle : la démarche du peintre autorise celle du cinéaste. L'inscription chez Bruegel de sujets bibliques dans le temps et l'espace contemporains les charge d'une efficace, d'une force tangible : ils deviennent partie constituante de la réalité. De même, lorsqu'il aborde le sujet mythologique de La Chute d'Icare, c'est sans solution de continuité que le tableau se fait attentif au réel et ouvert à la fiction. De ce mélange, de ces interactions, de cette manière d'envisager ce qu'on nomme le «réel», le film de Pazienza saura se souvenir, qui intègre et fait tenir ensemble cinéma du réel, cinéma de fiction. La fiction d'Ovide, certes, mais aussi bien les multiples fictions singulières, c'est-à-dire la manière dont nous - l'auteur lui-même, des inconnus, des voisins, des philosophes, des astronomes, des statisticiens, des écrivains, des hommes politiques, des chômeurs... donnons forme à chaque fois au monde dans lequel nous vivons. Le réel est aussi tissé de nos rêves : telle était la leçon du maitre flamand, que relève et prolonge à son tour le film de Pazienza. Mieux même : c'est peut-être à partir de nos rêves que l'on peut tenter de déplacer les termes de cette réalité. Car, si Icare tombe auprès du laboureur acharné à tracer les sillons de son propre labyrinthe, le souvenir du vol de Dédale - expressément convoqué dans le film autour de l'apocryphe du Musée Van Buuren - reste celui d'un vol parfaitement réussi.

Chez Bruegel, le monde ne s'ordonne pas uniquement à partir d'un point de vue central : la vision à vol d'oiseau est aussitôt complétée, ou contredite, par la multiplicité des scènes, des motifs, et l'attention extrême portée aux détails. Si la Renaissance fait de l'homme le centre de l'univers, et de ce dernier l'objet de sa vision, Bruegel place l'homme au centre de ses compositions, moins en situation assurée de maîtrise qu'en « objet » de recherche et de questionnement. Tout se passe comme si, dans ce XVIème siècle où les certitudes vacillent, où le système de Copernic reste une timide et scandaleuse hypothèse ${ }^{6}$, où le monde pourrait n'être plus à la mesure humaine, la peinture du Bruegel préfigurait déjà l'incertitude du paysage pascalien : «Une ville, une campagne, de loin c'est une ville et une campagne, mais à mesure qu'on s'approche... » ${ }^{7}$. Un siècle cependant sépare le peintre du philosophe - de l'ébranlement au basculement des certitudes - et si Pascal contemple avec effroi un univers infini, pour Bruegel, le monde n'est encore que grotesque: son scepticisme, parfois teinté d'humour, reste malgré tout un humanisme. 
6 Pour voir le monde, et l'homme en particulier, il faut s'en approcher : la vision des choses se fait au milieu des choses, le sujet du regard est nécessairement impliqué. Non cogitat qui non experitur était la devise des penseurs de ce siècle ${ }^{8}$; Pazienza reprend à son compte cette "frontalité », ce corps à corps avec le monde, qui caractérise à ses yeux la peinture flamande, et s'inscrit expressément dans ce qu'il nomme «cette école du regard ${ }^{9}$. La vision y reste une approche sensible, un « retour à un rapport essentiel au monde $»^{10}$, la vision d'un sujet pris lui-même dans le tissu du monde. Et c'est en apportant son propre corps à l'intérieur du film que Pazienza prolonge la leçon du peintre : un corps voyant et sensible - cette «sentinelle qui se tient silencieusement sous mes paroles et sous mes actes ", dont parle Merleau-Ponty ${ }^{11}$. Corps voyant, mais aussi bien visible, que le cinéaste nous présente avant même, mais précédant de peu, la première vision du tableau de Bruegel. Jean Renoir faisait, dit-on, ses films « avec la peau des choses ${ }^{12}$; c'est avec leur chair que Pazienza fait les siens. Le réel est d'abord présence charnelle, lesté qu'il est par le corps même du cinéaste. Retrouver le geste du peintre, qui donnait couleur, épaisseur, pesanteur même à chacun de ses personnages, implique aussi d'incarner Icare - de lui rendre son poids de chair, c'est-à-dire de réel - et de "greffer " sur son propre corps le tableau de Bruegel. Car si Bruegel parcourt la Belgique du XVIème siècle les deux pieds fermement plantés sur cette terre, Pazienza n'est belge que par « accident » et le sol est singulièrement mal assuré sous ses pas : face à un réel qui se dérobe - ou trop proche pour pouvoir le nommer -, ce corps reste la seule certitude d'habiter un monde, où la matière et l'homme lui-même se diluent en probabilités.

7 «Bruegel a peint beaucoup de choses qu'on ne peut peindre », écrivait son ami Abraham Ortelius, ajoutant aussitôt : "Il donne souvent à comprendre au-delà de ce qu'il peint » ${ }^{13}$. Paysage avec la chute d'Icare pose la question de notre regard sur l'image, mais également sur le monde visible. Ce tableau où l'œil est invité à cheminer sans rupture du laboureur au pâtre rêveur, du navire à Icare et au pêcheur, autorise dans sa composition «les retours en arrière et la fantaisie des détours ${ }^{14}$; la splendeur du soleil couchant recouvre également la terre grasse entaillée de sillons et les jambes de l'homme qui s'en est arraché et qui se noie. Cette image, parce qu'elle est « indécidable $»^{15}$, attend de son spectateur qu'il lui donne un ou des sens multiples : elle est bien " offre faite au regard d'une liberté » ${ }^{16}$. Alors que, dans le récit d'Ovide, le laboureur, le berger, le pêcheur sont témoins de la chute, chez Bruegel, celle-ci passe inaperçue: au-delà du regard, du visible et de l'invisible, la question que pose ce tableau est bien celle de l'« invention " du spectateur. Car, si le tableau déplace les termes de la fable, c'est bien pour nous constituer en spectateurs - de l'image et du monde. Comme toute image authentique, ce tableau «fonde la condition de possibilité d'un rapport, celui de notre regard à un monde visible $»^{17}$.

8 De même qu'au thème de la Belgique vue à vol d'oiseau succèdent dans le film ses variations en multiples aspects, de même le regard, que Pazienza institue en sujet de son film, se divise aussitôt en une multiplicité de regards. Le tableau de Bruegel devient monnaie d'échange dans un curieux commerce des regards: en imprimant sur soi La Chute d'Icare, le cinéaste se fait image, en même temps qu'il prête son corps à cette image. «Qu'est-ce que vous voyez?» est la question qu'il pose à des yeux non prévenus - pour constater qu'on ne rend compte que de soi, indirectement, à travers cette reproduction : «Le paysage est trop beau pour mettre un laboureur là-dedans!»; les enfants aussi privilégient le paysage: "C'est la Turquie! Parce qu'en Turquie il y a beaucoup de moutons ». Personne ne voit Icare, ou le prend pour un nageur; plus avant, une image 
montrant un homme qui se noie laisse ses parents totalement indifférents. Ceux qui se noient comme Icare, comme lui n'ont plus de visage: les hommes dans la queue du bureau de chômage rejettent et l'image et l'échange. Ceux que la société refuse de voir et dont l'image suscite habituellement l'indifférence, ne peuvent s'instituer en spectateurs, c'est-à-dire en sujets d'un regard, privés qu'ils sont du regard de l'autre. La patience du réalisateur, qui continue pourtant de tourner, visera moins à nous montrer finalement leur image qu'à constituer, c'est-à-dire à rendre possible leur regard sur nous. Pour des yeux plus avertis, le tableau de Bruegel peut aussi se métamorphoser en programme politique : le Premier Ministre en tient de préférence pour le laboureur qui «a les pieds sur terre ", alors qu'Icare, somme toute, s'avère n'être qu'un amateur; le Président du Parti Socialiste, en revanche, veut « redonner leur place au rêve et à l'utopie », face au laboureur qui n'est qu'un « arpenteur de normes ».

9 Si l'image, de peinture ou de cinéma, peut, à certaines conditions, nous apprendre à voir le monde et les autres, Pazienza s'attache, avec l'acharnement du laboureur, à creuser en épaisseur, à enchevêtrer et finalement à faire lever un essaim de questions. Qu'est-ce que voir? Que voit-on de la place où l'on se trouve ? S'agit-il de voir ou de regarder? Voir estil un acte physiologique, un acte de pensée ou les deux à la fois? N'y aurait-il pas, précédant toute vision, un désir de voir essentiel, qui se conjuguerait avec le désir d'être vu ? Qu'est-ce qui échappe à notre regard ? Qu'en est-il du "pouvoir » des images, entre feinte et fiction? Qu'en est-il du pouvoir de notre regard sur les autres? Et finalement, puisque la vue est l'instrument privilégié de la connaissance, comment se déprendre de soi, afin de se laisser regarder par les choses - «afin qu'entre soi et le visible, les rôles inévitablement s'inversent » et que nos yeux deviennent « ce qui a été ému par un certain impact du monde ? ${ }^{18}$.

Or, laisser ouvertes ces questions nécessite d'envisager pour le film une forme capable de les laisser résonner, c'est-à-dire d'organiser leur cœxistence, afin qu'elles rebondissent les unes sur les autres, et que l'écho s'en prolonge dans l'esprit du spectateur. La lumière était dans le tableau de Bruegel ce "grand unificateur $»^{19}$ qui autorisait la proximité de motifs antinomiques - le réalisme du laboureur et le rêve d'Icare; le temps, celui d'un film en train de se faire, permettra à Pazienza de rassembler en une totalité ouverte la multiplicité et surtout la disparate des questions.

L'inscription de l'auteur à l'intérieur de chacun de ses films reste pour Pazienza une marque de son engagement politique ${ }^{20}$ - dans le sens d'un partage, d'une circulation, à partir de la mise en mouvement de l'émotion et de la pensée de son spectateur. La distance critique, introduite dans le film par la présence du réalisateur, autorise en effet le spectateur à « construire à son tour la distance d'où il voit et d'où il peut juger " ${ }^{21}$. Dans Tableau avec chutes cependant, l'auteur s'y donne plus précisément dans un contact intime avec l'objet même qu'il propose au regard de son spectateur : le film garde la trace de la main qui semble le façonner peu à peu sous nos yeux. Mains de l'offre et du don, omniprésentes dans le film, mains qui écrivent et produisent du texte, mains qui photographient et qui filment, mains qui touchent et se tendent, qui montrent, qui rembobinent ou font défiler les images, mains qui écartent et retiennent: dans ce film, toute image, c'est-à-dire toute pensée, est d'abord un travail de la main. La main peut seule assurer cette fragilité des images, qui n'est pas d'incertitude ni de certitude figée, mais reste l'étincelle qui nous fera à notre tour lever les yeux. Car l'image dans ce film est chargée de faire voir et non pas de montrer ${ }^{22}$. 
Le lieu clos de l'appartement, à quoi se ramènent toutes choses, devient dans la "fiction » du film l'atelier où ce dernier s'élabore, par couches sédimentées - de la confusion première, à la complexité. À ce titre, l'atelier peut également devenir microcosme : son apparent désordre est à l'image d'un monde dont l'aspect serait essentiellement grotesque, voire carnavalesque, au sens où Mikhaïl Bakhtine envisageait la vision et l'œuvre de Rabelais ou de Dostoïevski : un monde "où se gèrent les mésalliances ", un monde où « tout cœxiste tout en étant de natures très différentes $»^{23}$. Ici encore Pazienza épouse le regard de Bruegel, et l'atelier peut devenir le lieu d'une plus haute poésie : « Si je parle de poésie, c'est comme si l'on parlait d'un zoo dans lequel certaines espèces pourraient se reproduire entre elles, en dehors de notre capacité à imaginer qu'un rhinocéros pourrait faire l'amour avec un sanglier $\aleph^{24}$. Tableau avec chutes gère parfaitement ces mésalliances, avec brio, profondeur, espièglerie parfois, ou fausse naïveté : la «fiction» de l'atelier autorise la constitution d'un texte où résonnent plusieurs voix à la fois, où s'interpénètrent plusieurs couches de significations provenant de registres très différents, où images et sons entrent en coalescence.

Cependant, l'atelier se fait aussi bloc de temps singulier. Le geste du réalisateur qui ramasse et noue entre elles différentes lignes de temps - l'autrefois du tableau et la vie quotidienne, le temps vécu dans l'histoire et celui, réservé, suspendu, de la réflexion instaure une durée d'une épaisseur particulière. Car les images du présent, c'est-à-dire les rencontres, les événements perçus au travers des journaux, des livres et de la télévision, sont convoquées dans la film à la manière de citations: le passé composé presque constant de la voix-off tient à même distance et unifie toutes choses, puisque le présent lui-même s'y transforme en un passé récent. Cette voix, qui n'est pas exactement celle du réalisateur, mais celle de la réflexion, du dialogue avec un autre soi-même, crée ainsi l'espace intime, préservé de l'atelier, en même temps qu'elle instaure la distance à laquelle le regard sur les images peut et doit enfin se poser. Le «tu» de cette voix-off nous implique imperceptiblement: le «tu» devient l'opérateur subtil qui permet de reverser les mouvements de pensée du réalisateur jusque dans l'esprit du spectateur - à la manière de vases communicants. Et si l'on s'interroge sur le "présent» de Tableau avec chutes, il est assurément cet instant précis et précieux où tout se récapitule, où le cinéaste appelle à soi et fait revenir tous les éléments de son film, juste avant de le composer. Le temps de l'atelier est un temps ouvert, un temps qui n'est achevé; il reste gros d'une promesse: celle de l'œuvre à venir. Inclus nous-mêmes dans l'atelier, c'est à nous qu'appartient désormais la tâche d'élaborer, de constituer le film.

14 Ainsi, grâce à un double mouvement - mouvement de présence, mouvement de retrait Pazienza laisse vacante la place qu'il nous offre d'occuper dans ce film. Tableau avec chutes relève de ces œuvres rares où l'auteur rend possible l'échange, «car il ne nous a pas fait montre de son bien, mais du nôtre $»^{25}$. Et si, pour Francis Ponge, l'atelier est ce lieu énigmatique, sorte de local ou de bocal organique, «construit par l'individu lui-même pour s'y enclore longuement, sans cesser d'y bénéficier pour autant, par transparence, de la lumière du jour » - en un mot, « une sorte de cocon $»^{26}-$, celui que construit Pazienza n'a d'autre but que notre métamorphose : il nous fait advenir en sujets actifs du regard, en acteurs critiques face au visible du monde et des images - en un mot, il nous transforme en spectateurs. Et c'est bien achever la leçon du passé, celle de Pieter Bruegel et de $L a$ Chute d'Icare. 


\section{NOTES}

1. Walter Benjamin, Paris, Capitale du XIXème siècle, Le livre des passages, Paris, Cerf, 1989, p. 409 [K 2,3] traduit dans Françoise Proust, L'Histoire à contretemps, Le Temps historique chez Walter Benjamin, Paris, Cerf, 1994, p. 45.

2. Georges Didi-Huberman, Devant le temps, Paris, Minuit, 2000, p. 22.

3. Cité dans Philippe et Françoise Roberts-Jones, Pierre Bruegel l'Ancien, Paris, Flammarion, 1997, p. 277.

4. Voir Marguerite Yourcenar, Souvenirs pieux, Paris, Gallimard, 1974, p. 55.

5. Voir Philippe et Françoise Roberts-Jones, op. cit. p. 193.

6. Voir Marguerite Yourcenar, L'CEuvre au noir, Paris, Gallimard, 1968, p. 272.

7. Blaise Pascal, Pensées, Paris, Seuil, 1962, p. 53.

8. Voir Marguerite Yourcenar, op. cit., p. 304.

9. Claudio Pazienza, Rencontre «Son réel » aux Ecrans Documentaires, Arcueil, 4 novembre 2007.

10. Ibidem.

11. Maurice Merleau-Ponty, L'Eil et l'esprit, Paris, Gallimard, 1964, p. 13.

12. André Bazin, Jean Renoir, Paris, Gérard Lebovici, 1989, p. 80.

13. Cité dans Philippe Roberts-Jones, Bruegel, La Chute d'Icare, Fribourg, Office du Livre, 1974, p. 48.

14. Idem, p. 20.

15. Marie José Mondzain, Le Commerce des regards, Paris, Seuil, 2003, p. 10.

16. Idem, p. 154.

17. Marie José Mondzain, Homo Spectator, Paris, Bayard, 2007, p. 22.

18. Maurice Merleau-Ponty, op. cit., p. 31 et p. 26.

19. Philippe Roberts-Jones, op. cit., p. 26.

20. Claudio Pazienza, entretien réalisé par Georges Heck, Association Vidéo Les Beaux Jours, Strasbourg.

21. Marie José Mondzain, Homo Spectator, p. 92.

22. Voir idem, p. 92.

23. Claudio Pazienza, dans Comment filmer l'art? Paris, Addoc, 1998, p. 48.

24. Claudio Pazienza, entretien réalisé par Georges Heck, Association Vidéo Les Beaux Jours, Strasbourg.

25. Blaise Pascal, op. cit., p. 279.

26. Francis Ponge, L'Atelier contemporain, Paris, Gallimard, 1977, p. 4.

\section{AUTEUR}

\section{ROSE-MARIE GODIER}

Maître de Conférences, Université Paris X 